\title{
Mss51 deletion enhances muscle metabolism and glucose homeostasis in mice
}

\author{
Yazmin I. Rovira Gonzalez,, ${ }^{1,2}$ Adam L. Moyer, ${ }^{1,2}$ Nicolas J. LeTexier, ${ }^{1}$ August D. Bratti, ${ }^{1}$ Siyuan Feng, \\ Congshan Sun, ${ }^{1,3,4}$ Ting Liu, ${ }^{5}$ Jyothi Mula, ${ }^{1}$ Pankhuri Jha, ${ }^{1}$ Shama R. Iyer, ${ }^{6}$ Richard Lovering, ${ }^{6}$ \\ Brian O'Rourke, ${ }^{5}$ Hye Lim Noh, ${ }^{7}$ Sujin Suk, ${ }^{7}$ Jason K. Kim, ${ }^{7,8}$ George K. Essien Umanah,, \\ and Kathryn R. Wagner ${ }^{1,3,4}$ \\ 'The Hugo W. Moser Research Institute, Kennedy Krieger Institute, Baltimore, Maryland, USA. ${ }^{2}$ Cellular and Molecular \\ Medicine Graduate Program, ${ }^{3}$ Department of Neurology, ${ }^{4}$ Department of Neuroscience, and ${ }^{5}$ Division of Cardiology, \\ Johns Hopkins School of Medicine, Baltimore, Maryland, USA. ${ }^{6}$ Department of Orthopaedics, University of Maryland \\ School of Medicine, Baltimore, Maryland, USA. ${ }^{7}$ Program in Molecular Medicine, University of Massachusetts Medical \\ School, Worcester, Massachusetts, USA. ${ }^{8}$ Division of Endocrinology, Metabolism and Diabetes, Department of Medicine, \\ University of Massachusetts Medical School, Worcester, Massachusetts, USA.
}

Myostatin is a negative regulator of muscle growth and metabolism and its inhibition in mice improves insulin sensitivity, increases glucose uptake into skeletal muscle, and decreases total body fat. A recently described mammalian protein called MSS51 is significantly downregulated with myostatin inhibition. In vitro disruption of Mss51 results in increased levels of ATP, $\beta$-oxidation, glycolysis, and oxidative phosphorylation. To determine the in vivo biological function of Mss51 in mice, we disrupted the Mss51 gene by CRISPR/Cas9 and found that Mss51-KO mice have normal muscle weights and fiber-type distribution but reduced fat pads. Myofibers isolated from Mss51KO mice showed an increased oxygen consumption rate compared with WT controls, indicating an accelerated rate of skeletal muscle metabolism. The expression of genes related to oxidative phosphorylation and fatty acid $\beta$-oxidation were enhanced in skeletal muscle of Mss51-KO mice compared with that of WT mice. We found that mice lacking Mss51 and challenged with a high-fat diet were resistant to diet-induced weight gain, had increased whole-body glucose turnover and glycolysis rate, and increased systemic insulin sensitivity and fatty acid $\beta$-oxidation. These findings demonstrate that MSS51 modulates skeletal muscle mitochondrial respiration and regulates wholebody glucose and fatty acid metabolism, making it a potential target for obesity and diabetes.

Conflict of interest: The authors have declared that no conflict of interest exists.

Copyright: (c) 2019, American Society for Clinical Investigation.

Submitted: May 15, 2018 Accepted: September 11, 2019 Published: September 17, 2019.

Reference information: /CI Insight. 2019;4(20):e122247. https://doi.org/10.1172/jici. insight.122247.

\section{Introduction}

Obesity and obesity-related metabolic disorders, such as type 2 diabetes mellitus (T2DM), have reached epidemic proportions (1-6), making the genes that are associated with a lean phenotype in animals intriguing as possible therapeutic targets. Myostatin (Mstn), a TGF- $\beta$ family member, is expressed predominantly in skeletal muscle and at lower levels in adipose tissue. Genetic ablation or postnatal inhibition of myostatin is associated with increased muscle mass and decreased adipose tissue $(7,8)$. Since skeletal muscle plays a major role in metabolic rate and insulin-mediated glucose clearance, several studies have investigated the effects of genetic and pharmacological inhibition of myostatin in mouse models of obesity and T2DM (9-14). These studies found that lack of myostatin resulted in increased glucose uptake and insulin sensitivity in skeletal muscle of mice (15-17). Moreover, myostatin-null mice were resistant to diet-induce obesity and fat mass accumulation and prevented diabetes and hyperphagia $(10,12,13,18-20)$, making the inhibition of myostatin and its specific targets an attractive strategy to prevent diet-induced changes in body weight and body composition.

Transcriptional profiles of muscle from $\mathrm{Mstn}^{-/-}$mice or mice treated with a myostatin/activin inhibitor (ActRIIB-Fc) demonstrated that Mss51 mitochondrial translational activator, also known as zinc finger, MYND domain-containing 17 (Zmynd17), was one of the most significantly downregulated genes (7, 21). Similarly, other studies have shown downregulation of Mss51 in mice where myostatin was inhibited 
postnatally, via a neutralizing antibody or by transgenic expression of an inhibitory pro-peptide (22-24). Such consistent downregulation of Mss51 upon myostatin inhibition in skeletal muscle suggests that MSS51 might be an effector or downstream regulator of the myostatin signaling pathway.

The function of mammalian MSS51 is unknown, although a putative ortholog in yeast that shares $19 \%$ amino acid identity with mouse MSS51 localizes to the mitochondria and serves as coordinator of Cox1 synthesis and assembly (25-29). We have previously shown that Mss51 transcripts, like Mstn, are highly expressed in skeletal muscle of WT mice and that MSS51 protein localizes to the mitochondria of human skeletal muscle (30). Disruption of the Mss51 locus in a mouse myoblast cell line (specifically, disruption of the conserved MYND domain), indicated that this Mss51 gene did not regulate myoblast proliferation or differentiation in vitro. However, Mss51-disrupted myotubes displayed increased levels of cellular ATP, $\beta$-oxidation, glycolysis, and oxidative phosphorylation (30). Moreover, several genes involved in fatty acid metabolism were increased in Mss51-disrupted myotubes, and the marker of cellular energy AMP-activated protein kinase $(\mathrm{AMPK} \alpha)$, as well as its activated form phosphorylated AMPK $\alpha$, were increased in myotubes with reduced Mss51 expression. In view of the positive bioenergetic effects Mss51 disruption had in vitro, we hypothesized that inhibiting Mss51 in vivo would reveal improved glucose homeostasis, fatty acid metabolism, and mitochondrial respiration.

Here, we elucidate the function of mammalian Mss51 in metabolism by characterizing an Mss51-KO mouse with targeted deletion of the conserved MYND domain region (exons 2 and 3). We find that Mss51KO mice recapitulate many of the beneficial metabolic features of inhibiting or ablating Mstn, including enhanced glucose uptake in skeletal muscle and reduction in obesity and insulin resistance after a high-fat diet (HFD) challenge. These findings define the bioenergetic features of mammalian Mss51 and highlight its potential as a new target for the treatment of obesity and T2DM.

\section{Results}

Mss51 deficiency does not affect muscle mass but attenuates age-induced weight gain. Mss51 is expressed predominantly in skeletal muscle, with a minor fraction in liver, brown adipose tissue (BAT), and white adipose (WAT) (Figure 1A). To understand the role of Mss51 in whole-body metabolism, we disrupted the Mss51 gene using CRISPR/ Cas9 techniques (Supplemental Figure 1; supplemental material available online with this article; https://doi. org/10.1172/jci.insight.122247DS1). Homozygous Mss51-KO animals were viable and fertile when crossed with C57BL/6J mice and to each other. After approximately 6 months of age, Mss51-KO mice displayed a $9.5 \%$ reduction in whole-body weight relative to WT (Figure 1B). WAT and BAT were also significantly reduced respectively by $54 \%$ and $24 \%$ in Mss51-KO mice when compared with WT (Figure 1C). Muscle weights and tibial lengths were similar in both populations (Figure 1, D and E). Consistent with these results, quantitative nuclear magnetic resonance (NMR) showed significant $8.9 \%$ reduction in total body weight and $40 \%$ reduction in fat mass in Mss51-KO mice compared with WT, while the lean mass and food intake was unchanged between groups (Figure 1, F-H). Serum levels of triglycerides and cholesterol did not change between groups (Figure 1I). Taken together, these results suggest that the reduction in body weight was due primarily to a lower fat mass

Genome-wide expression profiling of Mss51-KO and WT mice showed differential expression of genes related to oxidative phosphorylation and fatty acid $\beta$-oxidation. To explore the potential transcriptional targets involved in the Mss51-KO-mediated metabolic phenotype, the transcriptional profiles of the quadriceps muscle from Mss51-KO and WT mice were examined by RNA sequencing (RNA-seq). We observed 232 statistically significant differentially expressed genes between Mss51-KO and WT, with fragments per kilobase of transcript per million (FPKM) of 5 or greater for either group. Quadriceps muscles from mice deficient for the Mss51 gene showed both up- (158 genes) and downregulation (74 genes) of genes relative to control mice. To determine biological pathways influenced by Mss51 gene deficiency, we analyzed the 232 differentially expressed genes with an FPKM of at least 5 for either group using Ingenuity Pathway Analysis (IPA) software. The top 10 significantly affected canonical pathways in Mss51-KO mice are shown in Table 1. Genes involved in oxidative phosphorylation and fatty acid $\beta$-oxidation were among the top 2 differentially regulated pathways in Mss51-KO mice (Table 1). The FPKM abundance and variation of 33 genes corresponding to the top 10 significantly affected canonical pathways in Mss51-KO and control mice were examined. Specifically, expression of genes known to be important for mitochondrial function and fatty acid metabolism were significantly upregulated in the quadriceps of Mss51-KO mice (Figure 2A). Thus, both oxidative phosphorylation and fatty acid metabolism appear to be transcriptional targets implicated in the metabolic phenotype seen in Mss51-KO mice. 
A Mss51 mRNA expression in WT tissues

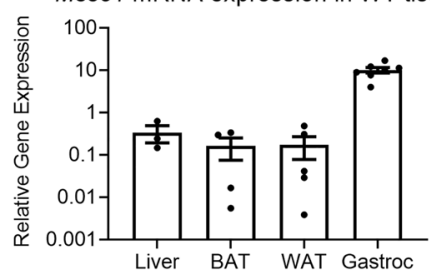

Liver BAT WAT Gastroc
B

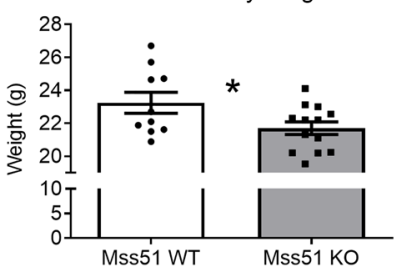

C

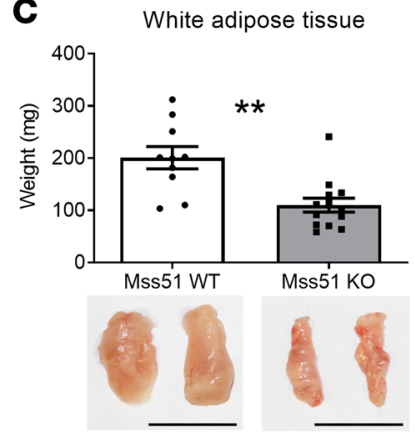

F
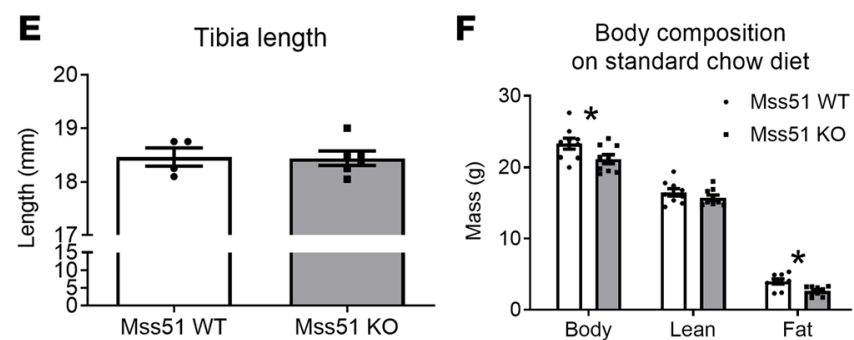

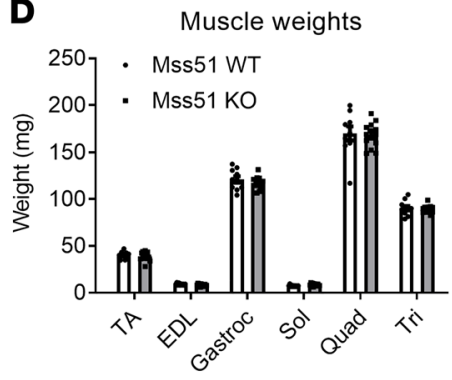

H

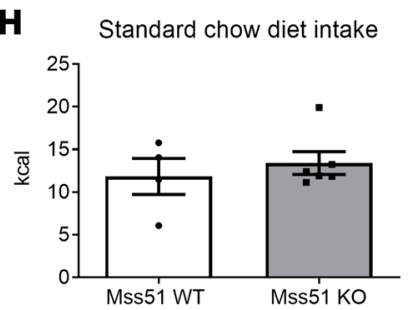

I

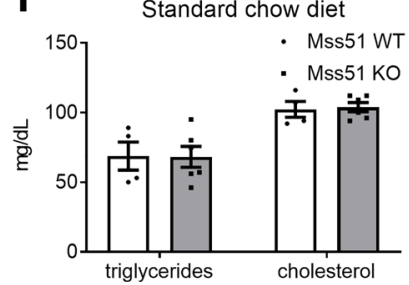

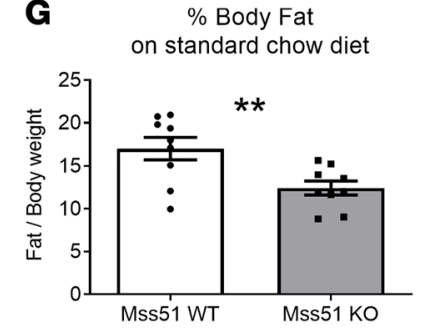

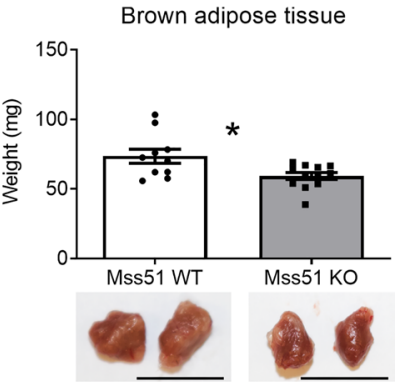

G

Figure 1. Mss51-KO animals have reduced fat pad mass and body weight but equal muscle mass when compared with Mss51-WT animals. (A) Relative Mss51 gene expression in metabolically active tissues of WT animals. Normalized to Tbp, Tfam, and B2m. BAT, brown adipose tissue; WAT, white adipose tissue; Gastroc, gastrocnemius muscle $(n=3-7)$. (B) Whole-body weight, (C) WAT and BAT weights with representative images, (D) muscle tissue weights, and (E) tibia length of 6-month-old female mice weaned onto a standard diet. (F) Body composition by quantitative NMR, (C) body fat normalized to body weight, and (H) food intake. (I) Triglycerides and cholesterol levels in serum ( $n=4-13$ per group, all females). Animals were sex and age matched. All data are expressed as mean \pm SEM. ${ }^{*} P<0.05 ;{ }^{*} P<0.01$ by Student's $t$ test. Scale bars: $0.88 \mathrm{~cm}$.

To validate our RNA-seq results, we selected 33 genes from the significantly differentially expressed 232-gene list, analyzed their expression levels with quantitative PCR (qPCR), and compared their qPCR fold-change expression with those obtained by RNA-seq (Supplemental Table 2). For qPCR, expression levels of all 33 genes were normalized with 2 different housekeeping genes (Tbp and Tfam) confirmed to be stably expressed in both mouse groups. Except for the genes Mdh1 and Ndufb10, which showed opposing trends, the fold-change expression of genes analyzed by RNA-seq correlated with the qPCR results (Supplemental Table 2). Accordingly, the positive Pearson's correlation coefficient between the 2 data sets was $r=0.89\left(P<0.0001, R^{2}=0.79\right)$, suggesting statistically significant similarities in direction of fold change determined by the RNA-seq and qPCR (Supplemental Figure 2).

Mss51-KO mice have increased mitochondrial respiration but not biogenesis. To investigate the effects of Mss51 deletion on mitochondrial respiratory capacity, we measured the oxygen consumption rate (OCR) in intact flexor digitorum brevis (FDB) fibers of 6-week-old Mss51-KO and WT mice. OCR was assessed before and after the addition of inhibitors to obtain several parameters of mitochondrial respiration (Figure 2B). Oligomycin, carbonyl cyanide- $p$-trifluoromethoxy phenylhydrazone (FCCP), and rotenone/antimycin A were consecutively added to the fibers to inhibit complex $\mathrm{V}$, uncouple the proton gradient, and inhibit complexes I and III, respectively. Myofibers isolated from Mss51-KO mice had significantly increased mitochondrial respiratory capacity compared with WT at both basal and maximal conditions (Figure 2, B and C). Moreover, we found a significant increase in proton leak and a trend toward increased spare respiratory consumption, but no change in ATP production (Figure 2C). This is consistent with previous in vitro results of 
Table 1. Top 10 canonical pathways and their genes differentially affected by genetic deletion of Mss51

\begin{tabular}{|c|c|c|c|}
\hline Pathway & Ratio & $P$ Value & Genes \\
\hline Oxidative Phosphorylation & $7.3 \%$ & $1.12 \times 10^{-5}$ & $\begin{array}{c}\text { Atp5g1, Atp5g3, Cox6b1, Cox7a1, Cox7a2, } \\
\text { Ndufb3, Ndufb7, Ndufb10 }\end{array}$ \\
\hline Mitochondrial Dysfunction & $5.3 \%$ & $4.56 \times 10^{-5}$ & $\begin{array}{l}\text { Atp5g1, Atp5g3, Cox6b1, Cox7a1, Cox7a2, } \\
\text { Ndufb3, Ndufb7, Ndufb10, Snca }\end{array}$ \\
\hline Ketolysis & $30 \%$ & $1.02 \times 10^{-4}$ & Bdh1, Hadhb, Oxct1 \\
\hline Sirtuin Signaling Pathway & $3.4 \%$ & $5.11 \times 10^{-4}$ & $\begin{array}{l}\text { Acadl, Atp5g1, Got2, Idh2, Ldhb, Ndufb3, } \\
\text { Ndufb7, Ndufb10, Pck1, Tomm5 }\end{array}$ \\
\hline Cellular Effects of Sidenafil (Viagra) & $4.6 \%$ & $1.64 \times 10^{-3}$ & Acta2, Adcy2, Cacna1a, Myl2, Myl3, Prkar2b \\
\hline Aspartate Degradation II & $28.6 \%$ & $1.85 \times 10^{-3}$ & Got2, Mdh1 \\
\hline CDK5 Signaling & $5.1 \%$ & $2.62 \times 10^{-3}$ & Adcy2, Cacna1a, Egr1, Ppp1r3c, Prkar2b \\
\hline
\end{tabular}

Ingenuity Pathway Analysis was performed on RNA-seq results from quadriceps muscle. All females, 6 months old, fed standard chow diet, $n=4$ for each group with significantly differentially expressed genes so at least one of the FPKM is $\geq 5$.

enhanced mitochondrial respiration in Mss51-disrupted myoblasts and suggests that Mss51 deletion enhances oxidative metabolism (30). Since mitochondrial proton leak has been associated with thermogenesis and standard metabolic rate (31-34), we tested body temperature at baseline and during acute cold challenge and found no significant differences in core temperature, food intake, $\mathrm{RER}, \mathrm{CO}_{2}$ production, and $\mathrm{O}_{2}$ consumption in 5-month-old Mss51-KO compared with WT mice (data not shown). Our data indicate that Mss51-KO mice are able to maintain their body temperature and metabolism at relatively similar levels as WT when challenged with acute cold temperatures.

To determine if Mss51 ablation increased mitochondrial biogenesis and morphology, ultrastructural and mitochondrial DNA/nuclear DNA ratio analyses were performed. Mss51 deficiency was associated with normal copy numbers (Figure 2D) and morphology of mitochondria (Figure 2E and Supplemental Figure 3). Indices of mitochondrial capacity and abundance, such as succinate dehydrogenase activity (Supplemental Figure 4A), citrate synthase activity (Supplemental Figure 4B), and Western blot of total mitochondrial OXPHOS complexes (Supplemental Figure 4C), were unchanged between Mss51-KO and WT mice. Taken together, these data suggest that Mss51 ablation enhances mitochondrial respiration but not through mitochondrial biogenesis.

Enhanced peak mitochondrial respiration in Mss51-KO fibers correlates with augmented running endurance. Since aerobic capacity (or oxidative metabolism) impacts cardiorespiratory fitness (35) and is an important determinant of distance-running performance (36), we carried out a running-endurance test in which Mss51-KO and WT mice were run to exhaustion on a treadmill. We found that the Mss51-KO mice ran for a longer time (Figure 3A) and distance (Figure 3B) on the treadmill when compared with Mss51 WT littermates. The endurance test performance seen in Mss51-KO mice was not due to a switch in fiber type in the muscle of these mice (Supplemental Figure 5) nor due to changes in liver glycogen (Figure 3C) or muscle glycogen levels (Figures 3D). This suggests that the increased running endurance of Mss51-KO mice occurs in parallel to the greater peak mitochondrial oxidative phosphorylation capacity seen in the myofibers of these animals.

To assess force production and whole-body movement, in vivo quadriceps muscle isometric force (in vivo maximal torque) and forelimb grip strength were tested in Mss51-KO and WT mice. We found that quadriceps maximal isometric torque normalized to quadriceps muscle mass was significantly elevated in Mss51-KO mice when compared with WT (Figure 3E). When the quadriceps maximal torque was normalized to body weight $(P=0.0261)$ or quadriceps muscle weight $(P=0.0274)$, the Mss51-KO mice remained significantly stronger than WT mice (data not shown). Forelimb grip strength normalized to body weight (Figure 3F) was also markedly increased in Mss51-KO mice as compared with WT. Taken together, these data suggest that the Mss51-KO animals are capable of running a longer time and distance due in part to increased quadriceps and forelimb muscle strength and enhanced bioenergetics.

Mss51-KO animals fed a low-fat diet have less body fat and exhibit enhanced metabolism. To investigate the metabolic outcomes of Mss51-KO mice, 8-week-old animals were subjected to a low-fat diet (LFD; $10 \% \mathrm{kcal}$ fat) for 13 weeks and then placed in metabolic chambers. Consistent with our normal-chow findings, the 
A

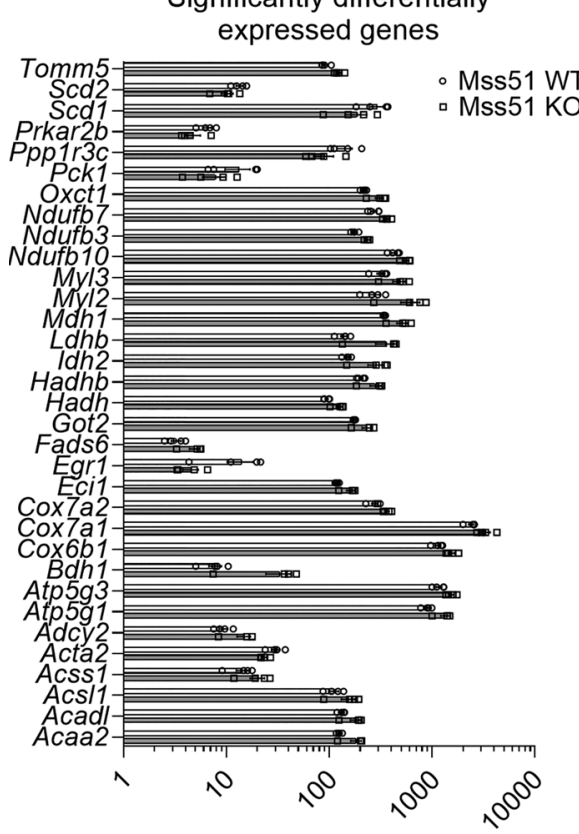

Expression $\left(\log _{10}\right.$ average FPKM)
B Mitochondrial Respiration in Myofibers

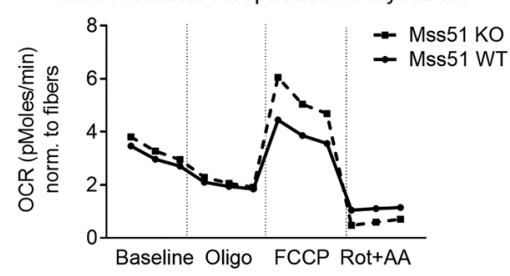

D

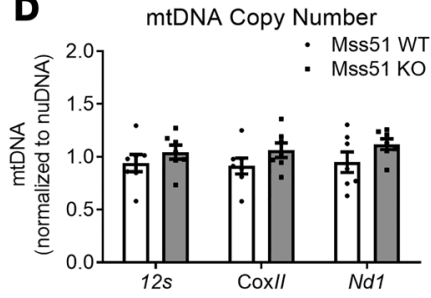

C Metrics of Mitochondrial Respiration
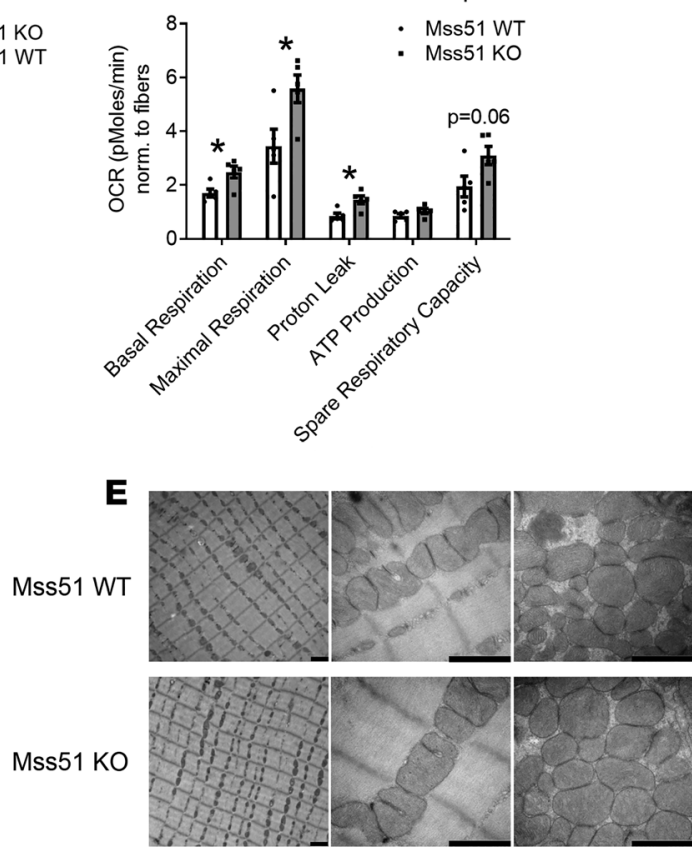

Figure 2. Mss51-KO mice show greater mitochondrial respiration but unchanged biogenesis. (A) Abundance of gene expression in quadriceps muscle of Mss51-KO and WT animals. Thirty-three genes corresponding to top 10 significantly affected canonical pathways with FPKM $\geq 5$ are shown. Analysis was done in quadriceps muscle of 6 -month-old mice on standard chow diet ( $n=4$ per group, all females). (B) Increased oxygen consumption rate in intact muscle fibers from Mss51-KO mice. Myofibers from the flexor digitorum brevis (FDB) muscle of Mss51-WT and Mss51-KO mice were isolated and subjected to bioenergetic analyses. Intact fibers were consecutively injected with pyruvate (baseline), oligomycin (Oligo), carbonyl cyanide $p$-(trifluoromethoxy)phenylhydrazone (FCCP), or rotenone/antimycin A (Rot+AA), and oxygen consumption rates (OCRs, pmol/min/fiber) were recorded. (C) Metrics of mitochondrial respiration as calculated from the bioenergetic analysis. All males, 6 weeks old, 1 reading per step (3 repeated measures per mouse fiber isolation, $n=5$ per group). (D) Mitochondrial gene copy number analysis in tibialis anterior muscle of 3-month-old Mss51-KO and WT mice normalized to nuclear DNA ( $n=7$ per group, all females). All data are expressed as mean \pm SEM. ${ }^{*} P<0.05$ by Student's $t$ test. (E) Electron microscopy of soleus muscle of 3-month-old female WT and Mss51-KO mice. Left panel magnification, $\times 9,700$ (scale bars $=50 \mathrm{~nm}$ ); middle and right panel magnification, $\times 50,000$ (scale bars $=500 \mathrm{~nm}$ ).

Mss51-KO animals were resistant to gaining weight upon LFD consumption. Specifically, Mss51-KO mice fed an LFD had a significant decrease in fat mass and a trend toward decreased body weight when compared with control animals (Figure 4A). Total lean mass remained unchanged between groups. Percentage body fat was significantly dampened in Mss51-KO mice compared with controls (Figure 4B). To further investigate the basis of reduction in body fat, LFD-fed Mss51-KO and WT animals were placed in metabolic chambers. Activity levels and respiratory exchange ratios were similar in Mss51-KO versus WT (data not shown). However, the $\mathrm{OCR}$ and $\mathrm{CO}_{2}$ production rates were significantly increased in Mss51-KO mice (Figure 4C), suggesting an increased metabolic rate. Similarly, the energy expenditure was also significantly increased in Mss51-KO mice, while food intake remained unaffected in both groups (Figure 4, C and D), implying that energy intake did not exceed expenditure, contributing to an enhanced resting metabolic rate. These data suggest that Mss51 deficiency has a role in regulating fat mass and energy production in mice.

Feeding Mss51-KO mice an HFD resulted in a obesity-resistant phenotype. To test whether Mss51 deficiency could ameliorate HFD-induced weight gain and hepatic steatosis, 8-week-old WT and Mss51-KO mice were fed an HFD ( $60 \% \mathrm{kcal}$ fat) for 13 weeks. Whole-body weight was significantly decreased in Mss51-KO animals when compared with WT littermates on HFD (Figure 5, A and B) and standard chow (Figure 5A). Mss51-KO mice showed significantly less change in body weight than HFD-fed WT when comparing the change in body weight from week 1 to week 13 (Figure 5C). The significant rise in body weight in HFD-fed WT mice was maintained until the end of the HFD challenge (Figure 5D). Like the standard-chow results, Mss51-KO mice had a significant reduction in WAT and BAT weight (Figure 5E). Moreover, HFD-fed Mss51-KO mice showed a significant reduction in whole-liver weight when compared with WT counterparts (Figure 5F). Given leptin's role in regulating energy balance, we examined serum leptin levels of HFD-fed Mss51-KO mice and found that they were significantly reduced when compared with WT (Sup- 

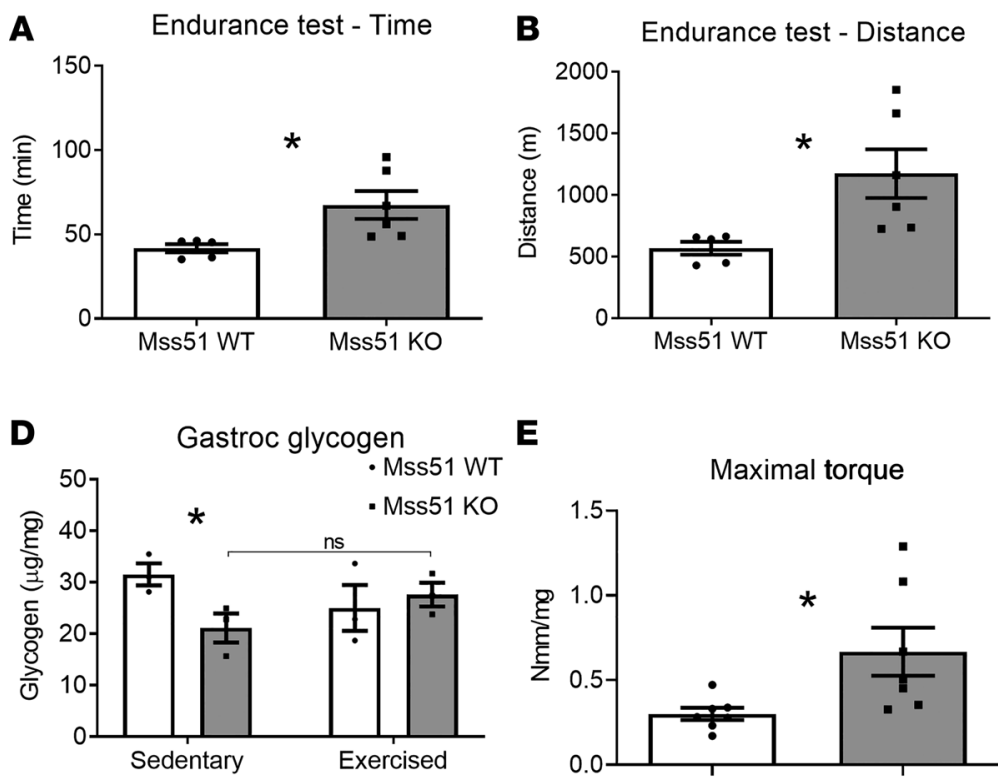

E

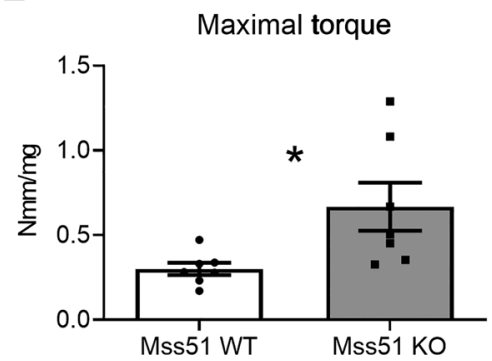

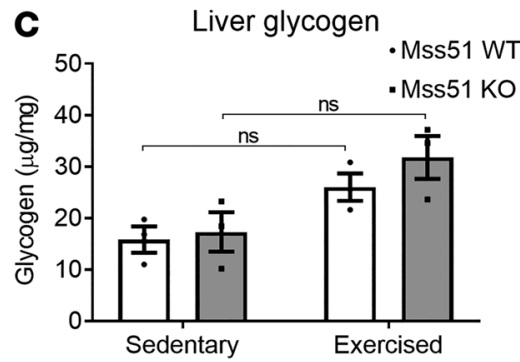

$\mathbf{F}$

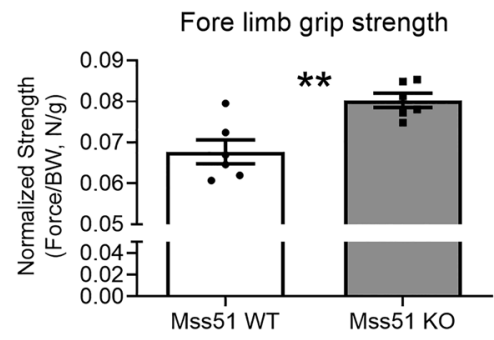

Figure 3. Enhanced running endurance of Mss51-KO mice when compared with WT. (A-D) Five-month-old mice were acclimated for 5 days and then ran to exhaustion on a horizontal treadmill starting at $6 \mathrm{~m} / \mathrm{min}$ with an increase of $2 \mathrm{~m} / \mathrm{min}$ every 5 minutes until mice reached exhaustion with the maximum speed reached set at $24 \mathrm{~m} / \mathrm{min}$. (A) Time and (B) distance to exhaustion. Glycogen levels in (C) liver and (D) gastrocnemius muscle of sedentary and exercised Mss51-KO and WT mice. ( $n=3$ per group, all females). (E) Maximal quadriceps isometric force (maximal in vivo torque) normalized to quadriceps mass, and (F) forelimb grip strength normalized to whole-body weight ( $n=6-7$ per group, all females). Animals were sex and age matched. BW, body weight. All data are expressed as mean \pm SEM. ${ }^{*} P<0.05 ;{ }^{* *} P<0.01$ by Student's $t$ test; $n s=$ not significant.

plemental Figure 6). Body composition analysis using quantitative NMR spectroscopy of WT and Mss51$\mathrm{KO}$ mice on HFD revealed a significant reduction in body mass, with a trend toward decrease in fat mass, but no change in lean mass or food intake of Mss51-KO mice as compared to WT (Figure 5, G and H). The tibia length and weights of different skeletal muscles at the end of the HFD challenge did not result in any significant difference between groups (Supplemental Figure 7). Therefore, reduced body weight in HFD-fed Mss51-KO mice can be attributed primarily to reduced fat and liver mass.

Since fatty acid $\beta$-oxidation was one of the most highly regulated pathways in our RNA-seq study and Mss51-KO animals accumulated less fat when challenged with an HFD, we tested the ability of these mice to oxidize radiolabeled $1-{ }^{14} \mathrm{C}$-oleic acid. Consistent with the reduced whole-body fat and body weight, Mss51-KO mice had a greatly increased ability to fully oxidize radiolabeled $1-{ }^{14} \mathrm{C}$-oleic acid in vivo (Figure $5 \mathrm{I}$ ). Because WAT weight was significantly reduced in Mss51-KO mice by $36 \%$, inguinal and epididymal histology was assessed. Mss51-KO inguinal and epididymal WAT contained smaller adipocytes than their WT counterparts (Figure 6, A and B) without significant changes in browning genes including Cidea, Ppara, Elovl3, and Ucp1 (data not shown). We also examined liver histology in HFD-fed Mss51-KO mice and saw less hepatic steatosis and lipid accumulation relative to WT controls (Figure 6C). Taken together, these data indicate that Mss51 deficiency helps prevent diet-induced obesity and fatty liver by upregulating fatty acid oxidation in adipose tissue.

Mss51-deficient mice are protected from HFD-induced insulin resistance. To uncover the effects of Mss51 deletion on glucose metabolism and insulin sensitivity in individual organs, we performed a 2-hour hyperinsulinemic-euglycemic clamp with ${ }^{3} \mathrm{H}$-glucose infusion and ${ }^{14} \mathrm{C}$-2-deoxyglucose injection in awake, 3-week-old Mss51-KO and WT mice weaned onto an HFD for 13 weeks (experiment done at 16 weeks of age). Body weight of Mss51-KO mice as determined by body composition analysis trended toward lower mass from weeks 10 to 13 of the HFD challenge when compared with WT controls (Figure 7A).

During the clamp, a $20 \%$ glucose solution was intravenously infused at variable rates to maintain euglycemia $(\sim 120 \mathrm{mg} / \mathrm{dL})$ in both groups of mice. Steady-state glucose infusion rates to maintain euglycemia were significantly increased by $45.5 \%$ in Mss51-KO animals versus WT, reflecting increased insulin sensitivity in Mss51-KO mice (Figure 7B). Insulin-stimulated whole-body glucose turnover was increased by 1.3 -fold $(P=0.092)$ in Mss51-KO mice, but whole-body glycogen plus lipid synthesis did not differ between groups (Figure 7, C and D). Whole-body glycolysis was significantly increased by $36 \%$ 
A Body composition on low-fat diet

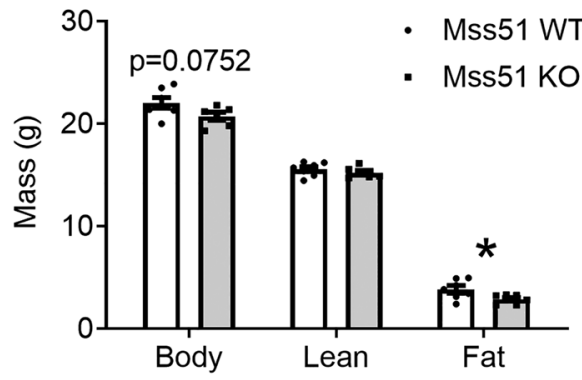

C $\mathrm{O}_{2}$ consumption and $\mathrm{CO}_{2}$ production

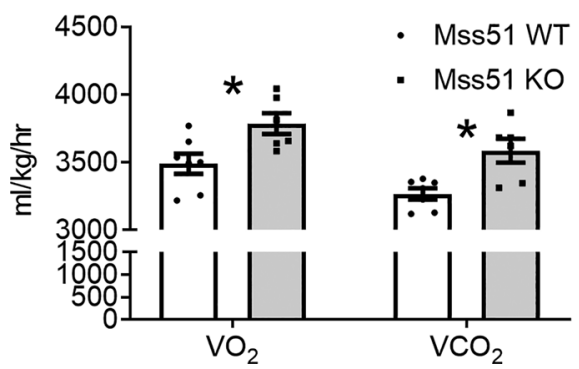

B $\quad \%$ Body fat on LFD

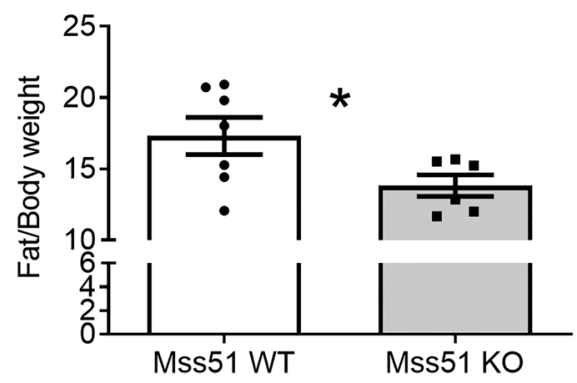

Energy expenditure

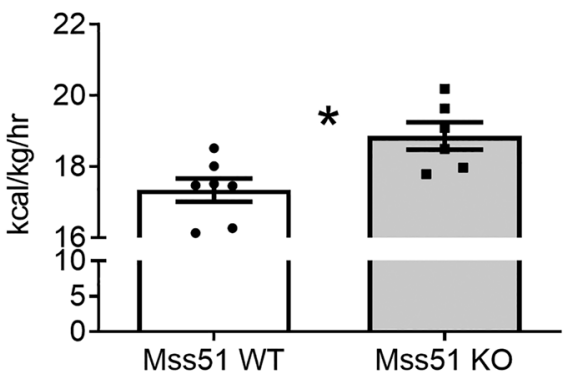

D

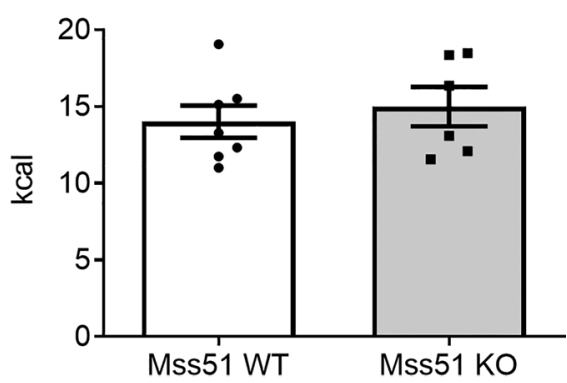

Figure 4. Metabolism of Mss51-KO mice when fed an LFD. (A and B) Body composition analysis and (C) indirect calorimetry and (D) food intake of mice when subjected to an LFD for 13 weeks ( $n=6-7$ per group, all females). All animals were age matched to those used for Figure 5 . Data are expressed as mean \pm SEM. ${ }^{*} P<0.05$ by Student's $t$ test.

in Mss51-KO mice compared with WT (Figure 7E). The basal plasma glucose levels after overnight fast tended to be lower in Mss51-KO mice (Figure 7F). These data indicate that HFD-fed Mss51-KO mice are more insulin sensitive than WT mice on HFD.

Increased insulin sensitivity in HFD-fed Mss51-KO mice was largely due to a significant 50\% increase in insulin-stimulated glucose uptake in skeletal muscle (gastrocnemius) (Figure 7G). Similarly, glucose uptake in WAT (epididymal fat) was significantly increased by $60.7 \%$ in Mss51-KO mice versus WT controls (Figure 7H). Basal hepatic glucose production (HGP) was not significantly altered between HFD-fed Mss51-KO and WT mice, but Mss51-KO mice showed a trend toward decreased HGP (1.7-fold reduction, $P=0.071$ ) during insulin clamp compared with WT mice (Figure 7I), suggesting insulin-mediated suppression of HGP in HFD-fed Mss51-KO mice. These data indicate that insulin resistance was selectively attenuated in skeletal muscle, WAT, and liver of HFD-fed Mss51-KO mice.

\section{Discussion}

In this study, we begin to characterize the biological function of mammalian Mss51 and explore the metabolic effects of its ablation in vivo. Mss51 is downregulated in the setting of myostatin and TGF- $\beta$ inhibition or IGF-1 stimulation in vitro (30). Unlike these modulators of muscle growth, knockout of Mss51 had no effect on muscle size or fiber type. This gives us a unique opportunity to separate out the metabolic effects of increased muscle from downstream effectors of these growth factors.

Mss51-KO mice displayed enhanced expression of both oxidative phosphorylation and fatty acid $\beta$-oxidation genes in skeletal muscle based on RNA-seq analysis (Table 1 and Supplemental Table 2). Moreover, Mss51-KO mice had a significant reduction in body weight, WAT, and BAT when compared with WT controls without changes in muscle weights, food intake, and tibia length (Figure 1). This lean phenotype was accentuated by challenging Mss51-KO mice with an LFD or HFD for 13 weeks. Mss51-KO mice were resistant to gaining weight upon either food challenge and had a decrease in fat mass and whole-body weight when compared with control animals (Figures 4 and 5). Upon placing LFD-fed Mss51-KO and WT animals in metabolic chambers, we saw that the oxygen consumption, carbon dioxide production, and energy expenditure were significantly increased in LFD-fed Mss51-KO mice (Figure 4), implying an enhanced resting metabolic rate and suggesting that Mss51 deficiency might have a role in regulating fat mass. 
A Weekly BW on chow or HFD

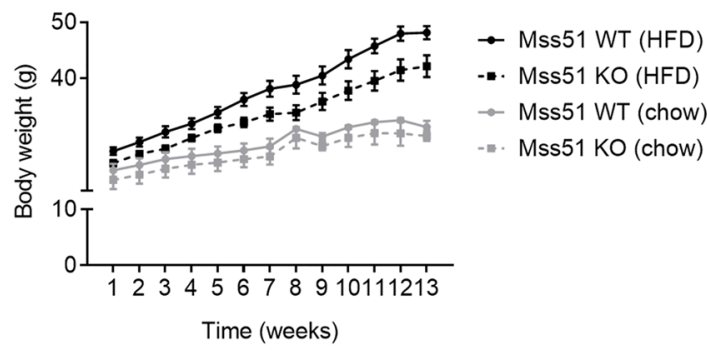

B

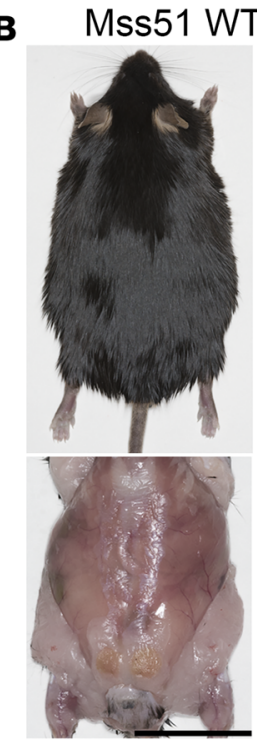

BAT

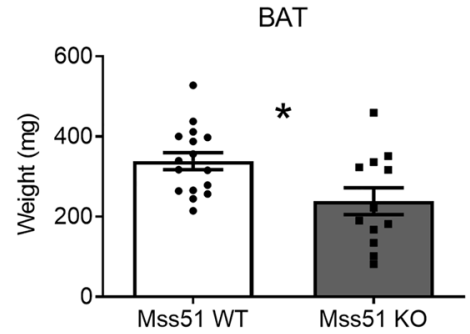

H

HFD food intake

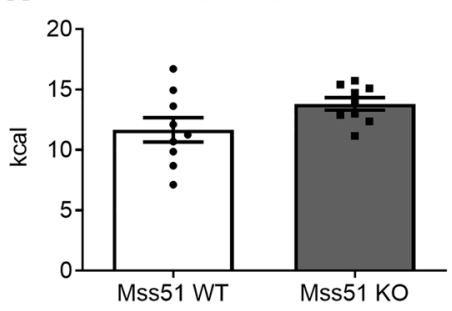

Mss51 KO

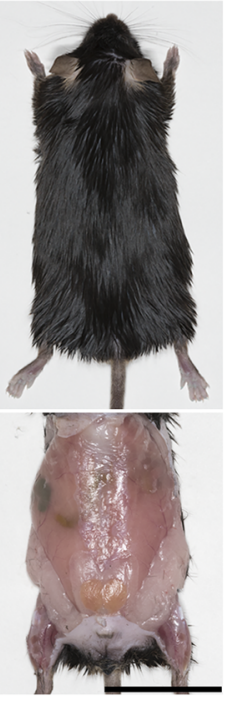

F
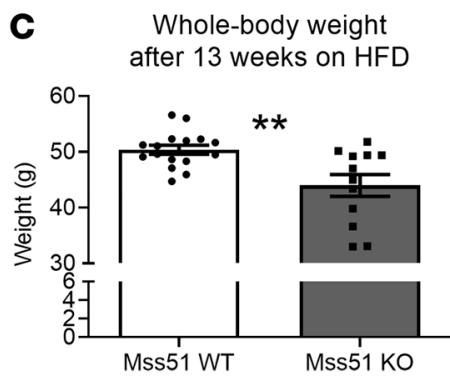

D

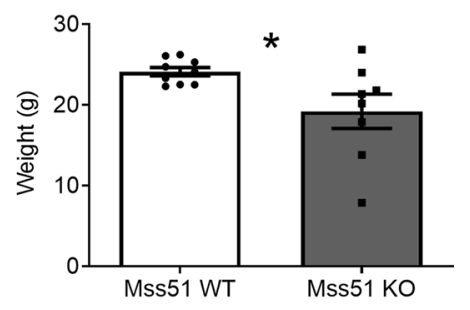

E

G Body composition on high-fat diet
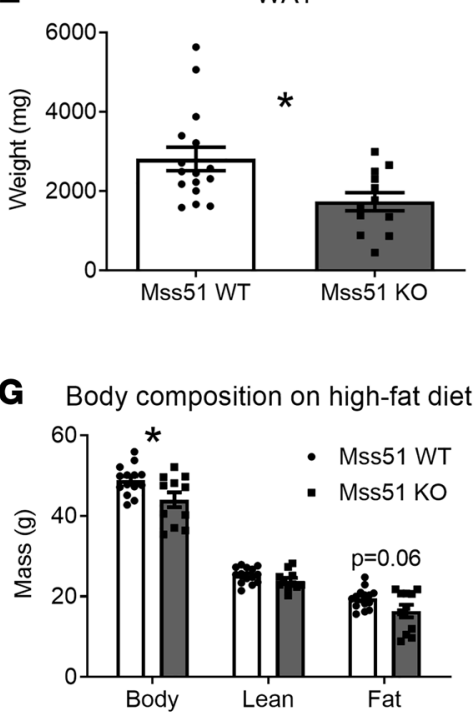

Figure 5. Mss51-KO mice are resistant to diet-induced obesity. (A) Weekly body weight (BW) on HFD or standard chow diet (chow) and (B) representative images of 2-month-old WT and Mss51-KO challenged with HFD for 13 weeks. (C) Whole-body weight and (D) change in weight from week 1 to week 13 on HFD (Delta BW). (E) WAT and BAT, and (F) whole-liver weights ( $n=8-16$, all males). Scale bars: $0.88 \mathrm{~cm}$. (G) body composition and (H) food intake of Mss51-KO and WT animals challenged with an HFD for 13 weeks ( $n=11-14$ per group, all males). ${ }^{*} P<0.05$; ${ }^{* *} P<0.01$ by Student's $t$ test. (I) In vivo fatty acid $\beta$-oxidation in mice fed an HFD for 13 weeks ( $n=4-5$ per group, all males). All mice were 2 months old before being placed on an HFD for 13 weeks. BW, body weight; WAT, white adipose tissue; BAT, brown adipose tissue. Animals were sex and age matched. Significance determined by 2-way repeated-measures ANOVA corrected for multiple comparisons using Sidak's test. ${ }^{* *} P<0.005$; ${ }^{* * *} P<0.0005$; ${ }^{* * *} P<0.0001$ by Student's $t$ test. All data are expressed as mean \pm SEM.

We investigated the in vivo mitochondrial respiratory capacity of Mss51-KO skeletal muscle and found that the OCR of myofibers isolated from Mss51-KO mice had significantly increased basal and maximal mitochondrial respiratory capacity compared with WT, with unchanged nonmitochondrial respiration, ATP production, and spare respiratory capacity between groups (Figure 2). Moreover, the remaining basal respiration not coupled to ATP production, or proton leak, was significantly increased in Mss51-KO myofibers when compared with WT mice. Increased mitochondrial respiratory capacity in Mss51-KO fibers was not due to mitochondrial capacity biogenesis (Figure 2). Consistent with this enhanced respiratory capacity, we found that the Mss51-KO mice ran for a longer time and distance than WT on a treadmill exhaustion test (Figure 3). These data are consistent with our previous in vitro results 
A

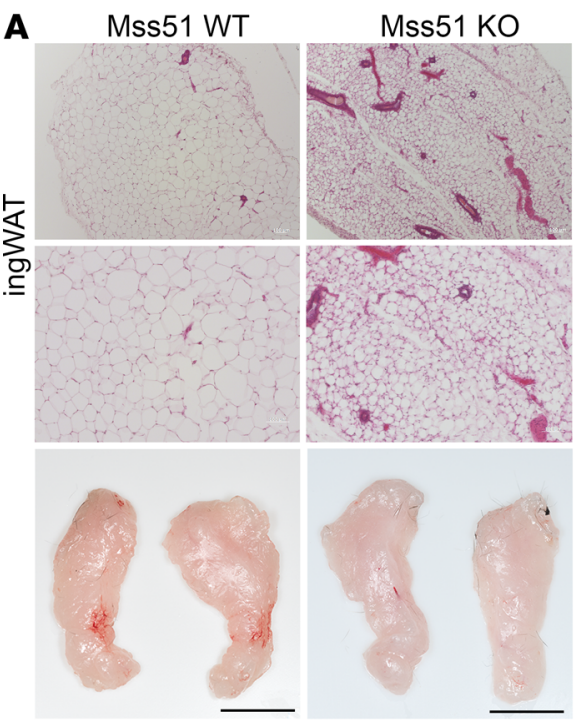

B

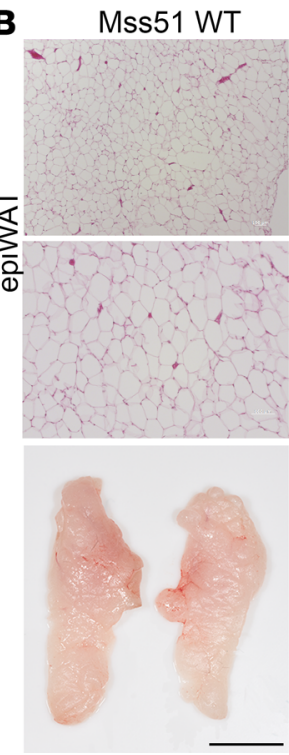

Mss51 KO
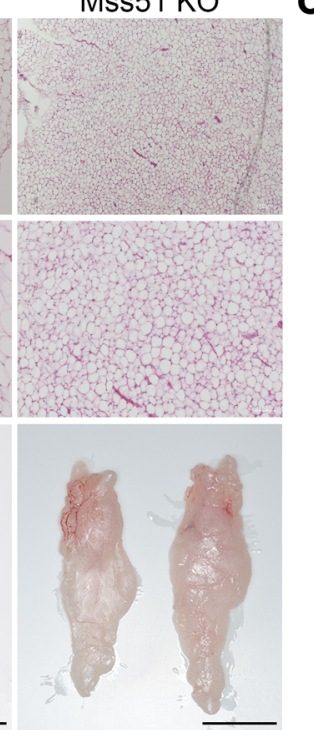
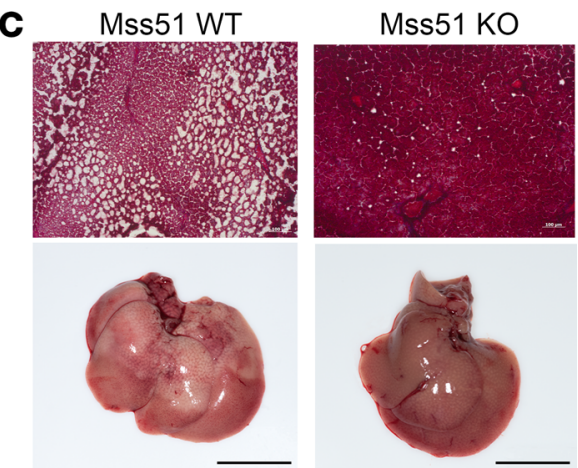

Figure 6. Adipose and liver histology of Mss51-KO and WT mice after HFD challenge. (A) Representative H\&E-stained sections and whole images of inguinal and (B) epididymal WAT from male mice on HFD for 13 weeks. Scale bars: $0.75 \mathrm{~cm}$. (C) Representative Masson's trichrome-stained sections and images of liver from male mice on HFD for 13 weeks. All mice were approximately 2 months old before being placed on a 13-week HFD challenge.

and suggest that Mss51 deletion enhances oxidative metabolism of skeletal muscle in vivo.

Skeletal muscle is the primary site of insulin-mediated glucose disposal, the largest reservoir of glycogen in the human body, and a key determinant of energy expenditure. Myostatin inhibition has been shown to greatly improve insulin sensitivity, increase glucose uptake, and decrease total body fat $(16,17,20,37)$. Constitutively active AMPK, exogenous administration of IGF-1, and TGF- $\beta$ inhibition also result in similar metabolic phenotypes (38-42). When subjecting our Mss51-KO mice to an HFD, we observed comparable effects to those of the myostatin-null mice. Mss51-KO body weight and adiposity were significantly reduced compared with WT after a chronic HFD challenge (Figure 5). Furthermore, HFD-fed Mss51-KO mice showed increased insulin sensitivity and enhanced glucose metabolism in skeletal muscle and WAT (Figure 7). Hepatic insulin action was also improved in HFD-fed Mss51-KO mice, and this is consistent with attenuated fatty liver in these mice (Figures 6 and 7).

Differing from the myostatin model, our mice did not show increased skeletal muscle mass (Figure 1). However, the lack of enhanced muscle mass in Mss51-KO animals is in agreement with the idea that hypermuscularity alone does not account for reduced adiposity and insulin sensitivity (16). Evidence reported here revealed that Mss51 can be inactivated to result in enhanced metabolic activity in both WAT and skeletal muscle without inducing hypertrophy.

Previously, Zhang and colleagues revealed elevated expression of key metabolic genes of fatty acid transport, higher rates of fatty acid oxidation, and browning of WAT in myostatin-null mice $(37,43)$. Similar to the effects of knocking out mouse myostatin on WAT, the Mss51-KO mice on HFD showed enhanced rates of fatty acid oxidation and also exhibited beneficial effects in adipose tissue, including less weight gain, reduced fat mass, improved glucose homeostasis, and lower levels of circulating leptin (Figures 5-7 and Supplemental Figure 6). However, we did not observe browning of WAT.

Recently, Fujita et al. (44) reported on the genetic deletion of Mss51 in mice and found that, similar to our results, Mss 51 deletion does not affect muscle mass and fiber types. However, they found mitochondria maintenance dysfunction in fast muscles in their mouse model as well as increased body weight, hepatic steatosis, glucose intolerance, and insulin resistance. These results are in contrast with the findings presented here, and are inconsistent with known effects of myostatin inhibition (which downregulates Mss51 in several models), including fat accumulation resistance and increased glucose uptake and insulin sensitivity in muscle $(7,12,17,20-24,45)$. The results presented here of enhanced mitochondrial respiration are also consistent with previously reported in vitro studies of Mss51 knockdown (30). It is possible that the difference in mouse model design between the Mss51-KO mouse model presented here and that reported by Fujita et al. is responsible for the phenotypic disparities between models. 
A

Body weight (weaned onto a HFD for 13 weeks)

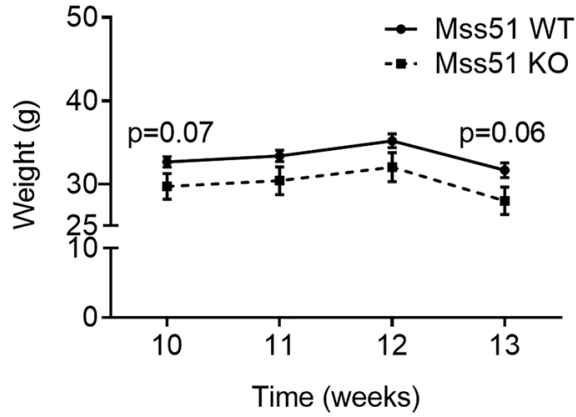

D Whole-body glycogen synthesis

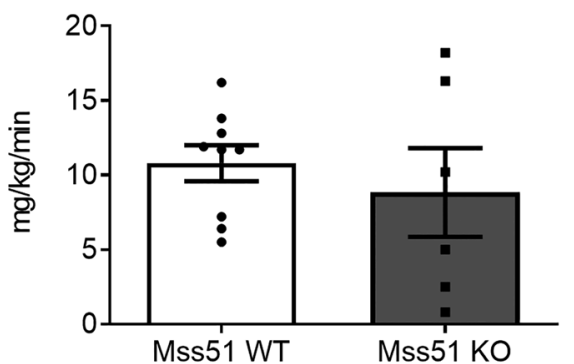

G

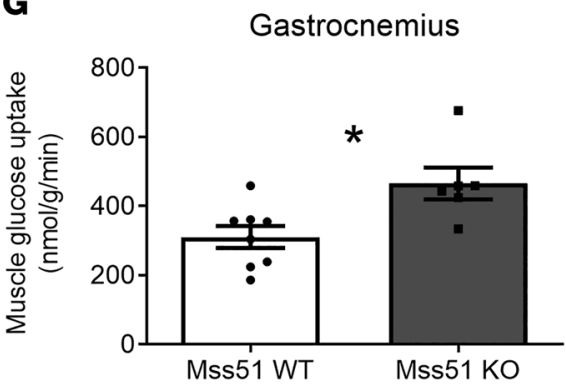

B
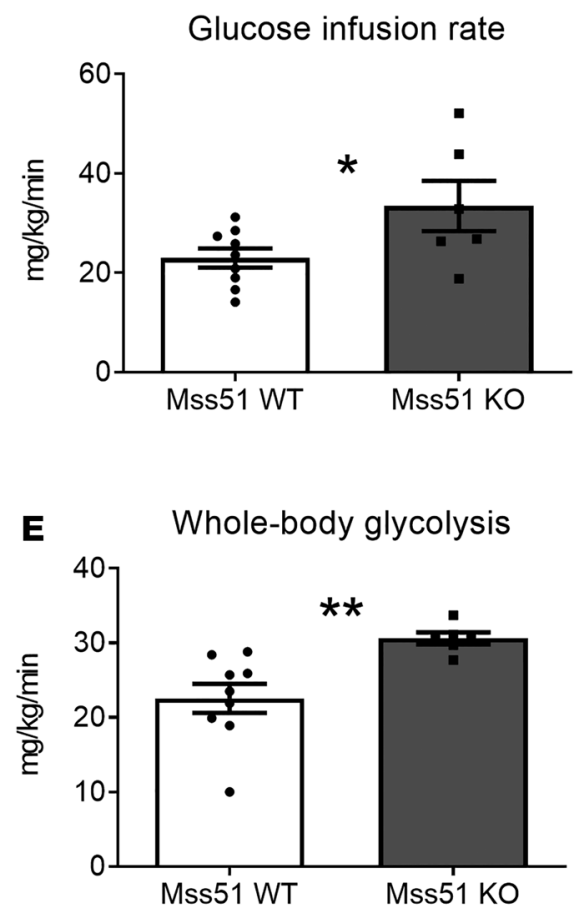

H

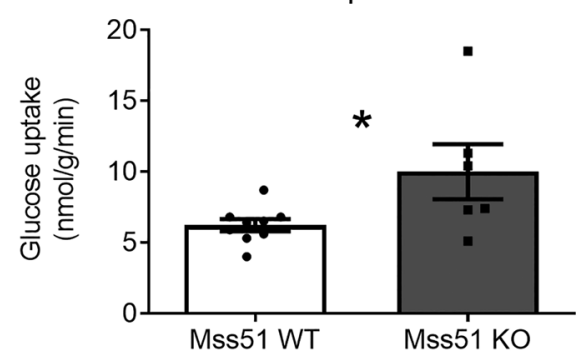

C

Whole-body glucose turnover
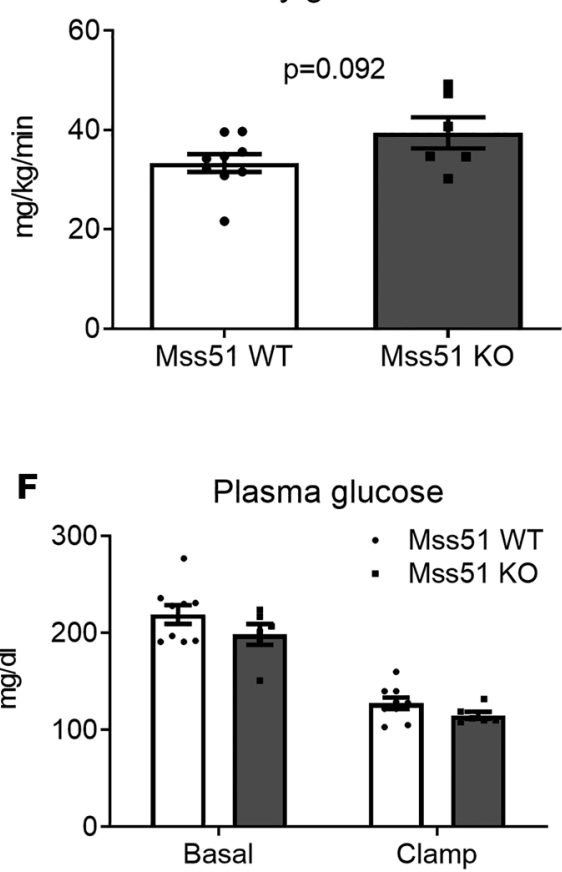

I

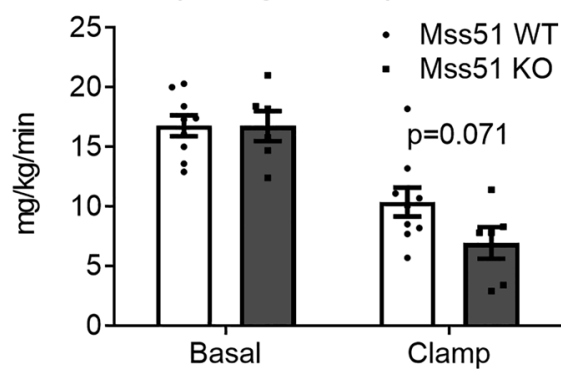

Figure 7. Mss51 deletion improves insulin sensitivity in mice with diet-induced obesity. Three-week-old Mss51-KO and WT male animals were weaned onto an HFD for 13 weeks and then hyperinsulinemic-euglycemic clamping on awake mice was performed. (A) Body weight, (B) steady-state glucose infusion rate, (C) whole-body glucose turnover, (D) glycogen synthesis, and (E) glycolysis of Mss51-KO versus WT mice. (F) Basal and clamped plasma glucose levels. Insulin-stimulated glucose uptake in (G) skeletal muscle (gastrocnemius) and (H) white adipose tissue (epididymis). (I) Basal and insulin-stimulated hepatic glucose production ( $n=6-9$ per group, all males). All animals were 3 weeks old before being weaned onto an HFD for 13 weeks. Animals were sex and age matched. Data are expressed as mean \pm SEM. ${ }^{*} P<0.05 ;{ }^{*} P<0.01$ by Student's $t$ test.

Ablation of Mss51 appears to enhance the metabolic state of skeletal muscle and adipose tissue, suggesting that Mss51 inhibition may confer beneficial effects on the metabolic state of skeletal muscle and adipose tissue in humans. Although the exact mechanism of Mss51 inhibition resulting in enhanced metabolism is unknown, our results suggest that it is not due to mitochondria biogenesis. Mss51 deletion appears to abrogate the accumulation of excess lipids and promote fatty acid oxidation in both skeletal muscle and WAT by maintained expression and activity of key mediators of fatty acid transport and oxidation in these tissues. Collectively, these in vivo data obtained in mice advocate further investigation and implicate Mss51 as a potential target for obesity and T2DM.

\section{Methods}

Animals. Both female and male mice of specified ages were maintained under a 12-hour light/12-hour dark schedule with ad libitum access to food and water. Inhalation overdose of isoflurane followed by cervical dislocation was the euthanizing method used in all experiments.

Generation of Mss51-KO mice (C57BL/6J background). Two CRISPR gRNAs targeting the first translat- 
ed exons (exons 2 and 3) of the Mss51 locus were utilized as depicted in Supplemental Figure 1A. These exons were targeted because they encompass the conserved MYND-type zinc finger domain. Selection was also based on CRISPRseek, scoring potential gRNAs to predict off-target effects. An injection mix carrying the Cas 9 mRNA and each gRNA at a ratio of 1:20 (ng/ $\mu \mathrm{L})$ was delivered to single-cell B6SJL/F2 embryos at the Johns Hopkins Transgenic Core Facility. After embryo implantation into pseudopregnant mice, founders were selected for backcrossing with C57BL/6J mice for 10 generations. Mss51 homozygous knockout (Mss51-KO) mice were obtained from mating of heterozygous siblings. Using either Platinum Pfx Polymerase (11708013, Life Technologies) or Taq Polymerase (18038042, Life Technologies), genotyping was performed by PCR with primers spanning both predicted cut sites, allowing for simple screening of deletions (Supplemental Figure 1B). Pfx Forward, 5'-GGGCCTTAGAGAGACTTGAATG-3'; Pfx Reverse, 5'-GCAGAGGCAGATGGATTTCT-3'; Taq Forward, 5'-GTCCCAGTTGATCCTCCAAA-3'; Taq Reverse, 5'-CGAAATCCAAAGCTTGATACTGG-3'. We isolated RNA from predicted Mss51-KO and WT skeletal muscle and performed real-time qPCR (RT-qPCR) of Mss51 (Supplemental Figure 1C). Additionally, RNA was isolated from Mss51-KO mice using the Direct-zol RNA MiniPrep kit (R2052, Zymo Research) and cDNA was synthesized using the SuperScript III First-Strand synthesis system with oligo-dT (18080051, Life Technologies). The cDNA was used as the template for PCR using primers flanking the deleted region (Forward, 5'-CTTGGAGGAAAGAGAAGGAAGG-3'; Reverse, 5'-TGAGGGATGCGAAAGGATTAG-3') and the resulting sequence was analyzed and compared to WT to ensure that the mRNA could not be translated to produce WT Mss51 protein. The mutant transcript omitted exons 2 and 3, which included the start codon, joining the 5' untranslated region (UTR) to exon 4 (Supplemental Figure 1A). The ExPasy translate tool (http://www.expasy.org) predicted that the first ATG codon after the UTR of the mutant transcript results in a short peptide of 11 residues followed by a stop codon. Upon obtaining RNA-seq data from quadriceps muscle of Mss51-KO and WT mice, we searched for the Mss51 locus and were able to confirm by RNA-seq that exons 2 and 3 were absent in Mss51-KO mice.

Single-fiber isolation and Seahorse OCR analysis. We isolated intact FDB fibers from the legs of 6-week-old ( $n$ $=6$ per group) Mss51-KO and WT mice by using an overnight treatment of collagenase A (10103578001, Sigma-Aldrich) at $1 \mathrm{mg} / \mathrm{mL}$ in Dulbecco's Modified Eagle's Medium (DMEM, Life Technologies) supplemented with $10 \%$ fetal bovine serum (Sigma-Aldrich) and $1 \%$ penicillin/streptomycin (Life Technologies). The OCR was measured using a Seahorse XF24 Flux Analyzer (Seahorse Bioscience). The day before the experiment, a 96-well plate was coated with polyethylenimine (1:15,000 dilution in distilled water; 408727, Sigma-Aldrich) and incubated overnight at $37^{\circ} \mathrm{C}$. Fibers were seeded at a density of 80-150 fibers per well in DMEM (11965118, Thermo Fisher Scientific). After centrifugation at $3000 \mathrm{~g}$ for 10 minutes, the DMEM was removed from each well and $180 \mu \mathrm{L}$ of assay buffer $\left(120 \mathrm{mM} \mathrm{NaCl}, 3.5 \mathrm{mM} \mathrm{KCl}, 1 \mathrm{mM} \mathrm{MgCl}, 0.4 \mathrm{mM} \mathrm{KH}_{2} \mathrm{PO}_{4}, 5 \mathrm{mM}\right.$ HEPES, $1.3 \mathrm{mM} \mathrm{CaCl}_{2}, 15 \mathrm{mM}$ glucose, and $5 \mathrm{mM}$ pyruvate; $\mathrm{pH} 7.4$ ) was added. After basal respiration was measured, $1 \mu \mathrm{M}$ oligomycin (Sigma-Aldrich), $1 \mu \mathrm{M}$ carbonyl cyanide $p$-(trifluoromethoxy)phenylhydrazone (FCCP) (Sigma-Aldrich), and $1 \mu \mathrm{M}$ rotenone and antimycin A (Sigma-Aldrich) were injected sequentially to each well. OCR per well was normalized to fiber number ( $\mathrm{pmol} / \mathrm{min} / \mathrm{single}$ fiber) using 3 independent measurements per mouse fiber isolation.

Body composition and indirect calorimetry. The body composition of Mss51-KO and WT littermates was compared by quantitative NMR (EchoMRI-100) performed at the Pathology Phenotyping Core at the Johns Hopkins University School of Medicine and using animals on standard chow, LFD, or HFD for indicated time points. Whole-body weight and whole-body fat and lean masses were measured noninvasively. Indirect calorimetry and energy balance parameters, including rates of $\mathrm{O}_{2}$ consumption $\left(\mathrm{VO}_{2}\right)$ and $\mathrm{CO}_{2}$ production $\left(\mathrm{VCO}_{2}\right)$, respiratory exchange ratio $\left(\mathrm{RER}=\mathrm{VCO}_{2} / \mathrm{VO}_{2}\right)$, energy expenditure (EE), food and water intake, and physical activity were assessed noninvasively for 5 consecutive days using metabolic cages (Oxymax, Comprehensive Lab Monitoring System, Columbus Instruments) at the Center for Metabolism and Obesity Research at the Johns Hopkins University School of Medicine. $\mathrm{VO}_{2}, \mathrm{VCO}_{2}$, and $\mathrm{EE}$ data were normalized to body mass. Physical activity was measured by infrared beam breaks in the metabolic chambers.

LFD and chronic HFD in mice. Eight-week-old female Mss51-KO and WT mice were fed an LFD (10\% kcal fat; D12450B, Research Diets) for 13 weeks ad libitum to perform body composition and indirect calorimetry tests and lastly harvest tissues for weights ( $n=6-7$ per group). Male Mss51-KO and WT littermates were either weaned onto an HFD (60\% kcal fat; D12492, Research Diets) for 13 weeks ( $n=6-9$ per group) or aged to 8 weeks before the 13-week HFD challenge ( $n=8-16$ per group). 
In vivo fatty acid $\beta$-oxidation. Eight-week-old control and Mss51-KO mice were fed an HFD for 13 weeks and then housed in individual metabolic cages and injected with $1 \mu \mathrm{Ci}$ of $1-{ }^{14} \mathrm{C}$ oleic acid. ${ }^{14} \mathrm{CO}_{2}$ was collected by continuous bubbling of the air from the metabolic cage through $1 \mathrm{~N} \mathrm{NaOH}$ after $15,30,90$, 180 , and 240 minutes ( $n=4-5$ per group). The investigator conducting the experiment was blinded to the genotypes of the mice.

EM, light microscopy, and fiber typing. EM was performed at the Johns Hopkins University School of Medicine Microscope Facility using extensor digitorum longus muscle sections. Individual muscles and fat pads were dissected on both sides of the body and weighed. Muscles and liver were frozen in cold isopentane (M32631, Sigma-Aldrich) and cryosectioned. Fat pads were fixed in 10\% zinc-formalin, embedded in paraffin, sectioned at $18 \mu \mathrm{m}$, and stained with hematoxylin and eosin (H\&E) or Masson's trichrome. For the fiber typing, the FDB muscle was stained with antibodies (all from the Developmental Studies Hybridoma Bank, University of Iowa, Iowa City, Iowa, USA) against type 1, 2a, and 2b fibers (BA.D5, 1:75 dilution; SC.71, 1:50 dilution; and BF.F3, 1:50 dilution, respectively). Fibers from entire FDB muscle were counted.

Citrate synthase and succinate dehydrogenase activity. Frozen sections of tibialis anterior muscle from 3-month-old Mss51-KO and WT female mice ( $n=7$ per group) on standard chow diet were stained for succinate dehydrogenase activity as previously described (46). For the citrate synthase activity assay, 2-3 mm of quadriceps from the same female cohort used in the succinate dehydrogenase activity assay were resuspended in CellLytic MT lysis buffer (Sigma-Aldrich; C3228), processed for protein isolation, and quantified using a BCA assay. Equal volumes of protein samples were plated in a 96-well plate in triplicate and activity reaction media (100 mM Tris-HCl, $1 \mathrm{mM}$ EDTA, $0.1 \mathrm{mM}$ Ellman's reagent, and $0.5 \mathrm{mM}$ acetyl-CoA; $\mathrm{pH} 8.1$ at $25^{\circ} \mathrm{C}$ ) was added to each well. The reaction was then started by adding $10 \mu \mathrm{L}$ of $10 \mathrm{mM}$ oxaloacetate to each as well and finally monitored at $412 \mathrm{~nm}$ until the absorbance rate plateaued. Only the linear portion of the curve was included in the data reduction and the pre-oxaloacetate rate was subtracted from the post-oxaloacetate rate to calculate citrate synthase activity.

Hyperinsulinemic-euglycemic clamp. This study was performed at the National Mouse Metabolic Phenotyping Center at UMass Medical School. Three-week-old Mss51-KO and WT male mice were weaned onto an HFD for 13 weeks. After 13 weeks of HFD, survival surgery was performed in Mss51-KO and WT mice at 5 to 6 days before clamp experiments to establish an indwelling catheter in the jugular vein. On the day of the clamp experiment, mice were fasted overnight ( $\sim 17$ hours), and a 2-hour hyperinsulinemic-euglycemic clamp was conducted in conscious mice with a primed and continuous infusion of human insulin (priming at $150 \mathrm{mU} / \mathrm{kg}$ body weight, followed by $2.5 \mathrm{mU} / \mathrm{kg} / \mathrm{min}$ Humulin [Eli Lilly]) (47). To maintain euglycemia, $20 \%$ glucose was infused at variable rates during the clamps. Whole-body glucose turnover was assessed with a continuous infusion of $\left[3-{ }^{3} \mathrm{H}\right]$ glucose (PerkinElmer), and 2-deoxy$\mathrm{D}-\left[1-{ }^{14} \mathrm{C}\right]$ glucose (PerkinElmer) was administered as a bolus $(10 \mu \mathrm{Ci})$ at 75 minutes after the start of the clamps to measure insulin-stimulated glucose uptake in individual organs. At the end of the clamps, mice were anesthetized and tissues were collected for biochemical assays as previously described (47).

Treadmill endurance. Exercise endurance test was performed using an Exer 6 rodent treadmill (Columbus Instruments). Five-month-old Mss51-KO and WT female mice were acclimated to the horizontal treadmill (no incline) for a 5 -minute $0 \mathrm{~m} / \mathrm{min}$ period, after which the speed was increased to $6 \mathrm{~m} / \mathrm{min}$ and raised by 2 $\mathrm{m} / \mathrm{min}$ every 5 minutes until $10 \mathrm{~m} / \mathrm{min}$ for a total of 5 acclimatization days. On the sixth day, mice ran on a horizontal treadmill starting at $6 \mathrm{~m} / \mathrm{min}$ with an increase of $2 \mathrm{~m} / \mathrm{min}$ every 5 minutes until they reached exhaustion (maximum speed set at $24 \mathrm{~m} / \mathrm{min}$ ), which was defined as 10 consecutive seconds on the shock pad a total of 3 times. Total time to exhaustion was recorded for each animal and the total distance to exhaustion was calculated. The investigator conducting experiment was blinded to the genotypes of the mice.

Glycogen levels. Gastrocnemius and liver tissues were isolated and snap frozen in isopentane from 5 -month-old sedentary and exercised female mice immediately after treadmill exhaustion $(n=3$ per tissue per group). Approximately $10 \mathrm{mg}$ of each tissue was homogenized rapidly and supernatant was prepared for colorimetric analysis of glycogen according to the manufacturer's instructions (ab169558, Abcam). Sample glycogen concentrations were measured and calculated against a glycogen standard curve.

In vivo isometric force. The femur of sedentary 5 -month-old mice ( $n=7$ per group) was stabilized and the ankle taped to a motor-driven lever arm $(48,49)$. The quadriceps muscle was stimulated by subcutaneous electrodes through the femoral nerve, resulting in knee extension. The lever arm was then used to force the knee joint into flexion. To fulfill 1 repetition, the quadriceps was stimulated for $200 \mathrm{~ms}$ to induce maximal isometric contraction. First and final repetition torque measurements were recorded and analyzed. 
RNA extraction and RT-qPCR. Cells and tissues were homogenized in TRIzol (15596018, Life Technologies) and total RNA was isolated using the Direct-zol RNA MiniPrep Kit (R2052, Zymo Research) and cDNA was synthesized from either 200 ng of RNA per sample using the iScript cDNA Synthesis Kit (1708891, BioRad). cDNA was diluted 1:10 and used as the template for real-time PCR using a CFX Connect Real-Time PCR Detection System (Bio-Rad) and SYBR Green PCR Master Mix (Life Technologies). The following cycle profile was used: 1 cycle at $50^{\circ} \mathrm{C}$ for 2 minutes, 1 cycle at $95^{\circ} \mathrm{C}$ for 2 minutes, and 45 cycles of $95^{\circ} \mathrm{C}$ for 15 seconds and $60^{\circ} \mathrm{C}$ for 1 minute. Relative gene expression was determined using the $\Delta \Delta \mathrm{Ct}$ method normalized to the indicated reference genes through the analytical software qbase+ (Biogazelle). At least 2 reference genes were included in each qRT-PCR experiment, and qbase+ was used to run the geNorm algorithm to select the most stable and suitable reference gene(s) for each experiment. qPCR primers (Supplemental Table 1) were designed using Primer-BLAST (http://www.ncbi.nlm.nih.gov/tools/primer-blast).

$R N A$-seq and analysis. Sequencing was performed at Novogene (University of California, Davis, California, USA) after RNA quality assessment using RNA isolated from quadriceps muscle of 6-month-old WT and Mss51-KO female mice as input material for library preparations. The captured library was sequenced on an Illumina HiSeq 4000 platform according to the manufacturer's instructions for paired-end 150-bp sequencing with sequence coverage of 50 million read-pairs. RNA-seq reads were aligned with TopHat (version 2.1.1, http://ccb.jhu.edu/software/tophat/downloads) using MM10 genome. Cufflinks (version 2.2.1, http://cole-trapnell-lab.github.io/cufflinks) was used to estimate the expression level for each gene based on reads that have been uniquely mapped to the genome. Cufflinks reported the abundance of a transcript in FPKM mapped reads. Only transcripts or genes that have an FPKM $\geq 5$ in at least one of the sets were used to select differentially expressed genes. The canonical pathways and genes differentially affected by Mss51 gene deletion were analyzed using IPA (QIAGEN Inc., http://www.qiagenbioinformatics.com/ products/ingenuity-pathway-analysis).

Data availability. The accession number for the RNA-seq data reported in this study is GSE134519.

Statistics. The data are presented as mean \pm SEM and all statistical analyses (2-tailed Student's $t$ tests unless otherwise indicated) were performed using GraphPad Prism (version 6.0) for Windows. Probability $(P)$ values less than $0.05\left(^{*}\right), 0.01\left(^{* *}\right)$, and $0.001\left(^{* * *}\right)$ were considered statistically significant.

Study approval. The Institutional Animal Care and Use Committee of the Johns Hopkins University School of Medicine approved the animal procedures performed in this study.

\section{Author contributions}

YIRG, ALM, and KRW contributed to the experimental design. YIRG, ALM, NJL, ADB, SF, and CS conducted mouse experiments and acquired, analyzed, and interpreted data. ADB did the glycogen assay and CS ran mice to exhaustion and harvested muscles. SF assisted during tissue harvests and quantified total protein expression of mitochondrial complexes. TL and BO performed muscle fiber Seahorse experiments and analyzed data. JKK, HLN, and SS performed the hyperinsulinemic euglycemic clamp experiments in mice and interpreted data. SRI and RL performed in vivo isometric force measurement experiments. JM did the fiber typing staining and analyses. PJ helped collect tissues and ran qPCR plates. GKEU provided invaluable insight on antibody design and recombinant protein production. YIRG, NJL, and KRW wrote the manuscript.

\section{Acknowledgments}

We are very thankful for the RNA-seq data analysis done by Corina Antonescu (Johns Hopkins University School of Medicine) and for the Ingenuity Pathway Analysis of the RNA-seq data provided by Alexis Norris (Kennedy Krieger Institute, Baltimore, Maryland). We thank Susan Aja (Center for Metabolism and Obesity Research, Baltimore, Maryland) for her assistance with indirect calorimetry and cold-challenge experiments, Christopher Ward (University of Maryland School of Medicine) for sharing his techniques on isolating intact single muscle fibers, Michael Wolfgang and Paul Watkins for assistance with fatty acid oxidation, and William Wong for helpful discussions. Part of this study was performed at the National Mouse Metabolic Phenotyping Center at UMass funded by NIH grant 5U2C-DK093000 (to JKK).

Address correspondence to: Kathryn R. Wagner, Center for Genetic Muscle Disorders, Kennedy Krieger Institute, 707 North Broadway, Baltimore, Maryland 21205, USA. Phone: 443.923.9525; Email: wagnerk@kennedykrieger.org. 
1. van Dieren S, Beulens JW, van der Schouw YT, Grobbee DE, Neal B. The global burden of diabetes and its complications: an emerging pandemic. Eur J Cardiovasc Prev Rehabil. 2010;17 Suppl 1:S3-S8.

2. Jaacks LM, Siegel KR, Gujral UP, Narayan KM. Type 2 diabetes: A 21st century epidemic. Best Pract Res Clin Endocrinol Metab. 2016;30(3):331-343

3. Tabish SA. Is diabetes becoming the biggest epidemic of the twenty-first century? Int J Health Sci (Qassim). 2007;1(2):V-VIII.

4. Pêgo-Fernandes PM, Bibas BJ, Deboni M. Obesity: the greatest epidemic of the 21st century? Sao Paulo Med J. 2011;129(5):283-284

5. Mitchell NS, Catenacci VA, Wyatt HR, Hill JO. Obesity: overview of an epidemic. Psychiatr Clin North Am. 2011;34(4):717-732.

6. GBD 2015 Obesity Collaborators, et al. Health effects of overweight and obesity in 195 countries over 25 years. N Engl J Med. 2017;377(1):13-27.

7. Welle S, Cardillo A, Zanche M, Tawil R. Skeletal muscle gene expression after myostatin knockout in mature mice. Physiol Genomics. 2009;38(3):342-350

8. McPherron AC, Lawler AM, Lee SJ. Regulation of skeletal muscle mass in mice by a new TGF-beta superfamily member Nature. 1997;387(6628):83-90.

9. Allen DL, Hittel DS, McPherron AC. Expression and function of myostatin in obesity, diabetes, and exercise adaptation. Med Sci Sports Exerc. 2011;43(10):1828-1835.

10. Hamrick MW, Pennington C, Webb CN, Isales CM. Resistance to body fat gain in 'double-muscled' mice fed a high-fat diet. Int J Obes (Lond). 2006;30(5):868-870.

11. McPherron AC, Guo T, Bond ND, Gavrilova O. Increasing muscle mass to improve metabolism. Adipocyte. 2013;2(2):92-98

12. Zhang $\mathrm{C}$, et al. Inhibition of myostatin protects against diet-induced obesity by enhancing fatty acid oxidation and promoting a brown adipose phenotype in mice. Diabetologia. 2012;55(1):183-193.

13. Guo T, Bond ND, Jou W, Gavrilova O, Portas J, McPherron AC. Myostatin inhibition prevents diabetes and hyperphagia in a mouse model of lipodystrophy. Diabetes. 2012;61(10):2414-2423.

14. Jiang JG, et al. Adeno-associated virus-mediated expression of myostatin propeptide improves the growth of skeletal muscle and attenuates hyperglycemia in $\mathrm{db} / \mathrm{db}$ mice. Gene Ther. 2017;24(3):167-175.

15. Dong J, Dong Y, Dong Y, Chen F, Mitch WE, Zhang L. Inhibition of myostatin in mice improves insulin sensitivity via irisin-mediated cross talk between muscle and adipose tissues. Int J Obes (Lond). 2016;40(3):434-442.

16. Guo T, Jou W, Chanturiya T, Portas J, Gavrilova O, McPherron AC. Myostatin inhibition in muscle, but not adipose tissue, decreases fat mass and improves insulin sensitivity. PLOS ONE. 2009;4(3):e4937.

17. Zhang C, et al. Myostatin-deficient mice exhibit reduced insulin resistance through activating the AMP-activated protein kinase signalling pathway. Diabetologia. 2011;54(6):1491-1501.

18. Jackson MF, et al. The aging myostatin null phenotype: reduced adiposity, cardiac hypertrophy, enhanced cardiac stress response, and sexual dimorphism. J Endocrinol. 2012;213(3):263-275.

19. Hjorth M, et al. Myostatin in relation to physical activity and dysglycaemia and its effect on energy metabolism in human skeletal muscle cells. Acta Physiol (Oxf). 2016;217(1):45-60.

20. McPherron AC, Lee SJ. Suppression of body fat accumulation in myostatin-deficient mice. J Clin Invest. 2002;109(5):595-601.

21. Rahimov F, et al. Gene expression profiling of skeletal muscles treated with a soluble activin type IIB receptor. Physiol Genomics. 2011;43(8):398-407.

22. Welle S, Burgess K, Mehta S. Stimulation of skeletal muscle myofibrillar protein synthesis, p70 S6 kinase phosphorylation, and ribosomal protein S6 phosphorylation by inhibition of myostatin in mature mice. Am J Physiol Endocrinol Metab. 2009;296(3):E567-E572.

23. Latres E, et al. Myostatin blockade with a fully human monoclonal antibody induces muscle hypertrophy and reverses muscle atrophy in young and aged mice. Skelet Muscle. 2015;5:34.

24. Miao Y, Yang J, Xu Z, Jing L, Zhao S, Li X. RNA sequencing identifies upregulated kyphoscoliosis peptidase and phosphatidic acid signaling pathways in muscle hypertrophy generated by transgenic expression of myostatin propeptide. Int J Mol Sci. 2015;16(4):7976-7994

25. Perez-Martinez X, Butler CA, Shingu-Vazquez M, Fox TD. Dual functions of Mss51 couple synthesis of Cox1 to assembly of cytochrome c oxidase in Saccharomyces cerevisiae mitochondria. Mol Biol Cell. 2009;20(20):4371-4380.

26. Ott M, Herrmann JM. Co-translational membrane insertion of mitochondrially encoded proteins. Biochim Biophys Acta. 2010;1803(6):767-775.

27. García-Villegas R, et al. The Cox1 C-terminal domain is a central regulator of cytochrome c oxidase biogenesis in yeast mitochondria. J Biol Chem. 2017;292(26):10912-10925.

28. Fontanesi F, Soto IC, Horn D, Barrientos A. Mss51 and Ssc1 facilitate translational regulation of cytochrome c oxidase biogenesis. Mol Cell Biol. 2010;30(1):245-259.

29. Soto IC, Fontanesi F, Myers RS, Hamel P, Barrientos A. A heme-sensing mechanism in the translational regulation of mitochondrial cytochrome c oxidase biogenesis. Cell Metab. 2012;16(6):801-813.

30. Moyer AL, Wagner KR. Mammalian Mss51 is a skeletal muscle-specific gene modulating cellular metabolism. J Neuromuscul Dis. 2015;2(4):371-385.

31. Jastroch M, Divakaruni AS, Mookerjee S, Treberg JR, Brand MD. Mitochondrial proton and electron leaks. Essays Biochem. 2010;47:53-67.

32. Thrush AB, Dent R, McPherson R, Harper ME. Implications of mitochondrial uncoupling in skeletal muscle in the development and treatment of obesity. FEBS J. 2013;280(20):5015-5029.

33. Sakellariou $P$, et al. Neuromuscular electrical stimulation promotes development in mice of mature human muscle from immortalized human myoblasts. Skelet Muscle. 2016;6:4.

34. Larrarte E, Margareto J, Novo FJ, Marti A, Alfredo Martínez J. UCP1 muscle gene transfer and mitochondrial proton leak mediated thermogenesis. Arch Biochem Biophys. 2002;404(1):166-171.

35. Aronow WS. Cardiovascular system. In: Birren JE, ed. Encyclopedia of Gerontology. 2nd ed. ScienceDirect; 2007:224-229.

36. Ghosh AK. Anaerobic threshold: its concept and role in endurance sport. Malays J Med Sci. 2004;11(1):24-36

37. Zhang $\mathrm{C}$, et al. Inhibition of myostatin protects against diet-induced obesity by enhancing fatty acid oxidation and promoting a 
brown adipose phenotype in mice. Diabetologia. 2012;55(1):183-193.

38. Koncarevic A, et al. A novel therapeutic approach to treating obesity through modulation of TGF $\beta$ signaling. Endocrinology. 2012;153(7):3133-3146.

39. Musi N, Goodyear LJ. Targeting the AMP-activated protein kinase for the treatment of type 2 diabetes. Curr Drug Targets Immune Endocr Metabol Disord. 2002;2(2):119-127.

40. Musi N. AMP-activated protein kinase and type 2 diabetes. Curr Med Chem. 2006;13(5):583-589.

41. Yakar S, et al. Liver-specific igf-1 gene deletion leads to muscle insulin insensitivity. Diabetes. 2001;50(5):1110-1118.

42. Aguirre GA, De Ita JR, de la Garza RG, Castilla-Cortazar I. Insulin-like growth factor-1 deficiency and metabolic syndrome. $J$ Transl Med. 2016;14:3.

43. Lebrasseur NK. Building muscle, browning fat and preventing obesity by inhibiting myostatin. Diabetologia. 2012;55(1):13-17.

44. Fujita R, et al. Zmynd17 controls muscle mitochondrial quality and whole-body metabolism. FASEB J. 2018;32(9):5012-5025

45. Zhao B, Wall RJ, Yang J. Transgenic expression of myostatin propeptide prevents diet-induced obesity and insulin resistance. Biochem Biophys Res Commun. 2005;337(1):248-255.

46. Carson FL, Cappellano CH. Histotechnology: A Self Instructional Text. 4th ed. Chicago, Illinois, USA: American Society for Clinical Pathology; 2014

47. Kim JK. Hyperinsulinemic-euglycemic clamp to assess insulin sensitivity in vivo. Methods Mol Biol. 2009;560:221-238.

48. Iyer SR, Valencia AP, Hernández-ochoa EO, Lovering RM. In vivo assessment of muscle contractility in animal studies. In: Kyba, M, ed. Skeletal Muscle Regeneration in the Mouse: Methods and Protocols. New York, New York, USA: Springer; 2016:293-307.

49. Pratt SJP, Shah SB, Ward CW, Inacio MP, Stains JP, Lovering RM. Effects of in vivo injury on the neuromuscular junction in healthy and dystrophic muscles. J Physiol (Lond). 2013;591(2):559-570. 